\title{
The Boolean SATisfiability Problem in Clifford algebra
}

\author{
Marco Budinich \\ University of Trieste and INFN, Trieste, Italy \\ mbh@ts.infn.it
}

September 23, 2018

\begin{abstract}
We present a formulation of the Boolean Satisfiability Problem in spinor language that allows to give a necessary and sufficient condition for unsatisfiability. With this result we outline an algorithm to test for unsatisfiability with possibly interesting theoretical properties.
\end{abstract}

\section{Introduction}

In 1913 Élie Cartan introduced spinors [3] and, after more than a century, this field still yields rich harvests. Spinors were later thoroughly investigated by Claude Chevalley [4] in the mathematical frame of Clifford algebras where they were identified as elements of Minimal Left Ideals of the algebra.

In this paper we establish a link between the famous Boolean Satisfiability Problem (SAT) and the algebra of fermions: we exploit the properties of Clifford algebra to give an algebraic formulation of SAT that yields a necessary and sufficient condition for unsatisfiability. This is not the first time that physics meets SAT: also statistical mechanics methods applied to SAT have obtained considerable successes [1].

In section 2 we succinctly resume basic properties of SAT and in section 3 we introduce an Abelian subalgebra of Clifford algebra and show that any Boolean expression is neatly represented by an idempotent of this subalgebra. In following section 4 we apply this method to transfer SAT problems in Clifford algebra and in section 5 we exploit this formulation to obtain, with a basic theorem of Clifford algebra, a necessary and sufficient condition for a SAT problem to be unsatisfiable. In final section 6 we use this result to outline an actual algorithm for SAT problems that turns out to be a relaxation of the standard Davis Putnam recursive algorithm [6].

For the convenience of the reader we tried to make this paper as elementary and self-contained as possible. 


\section{Satisfiability problem}

The Boolean Satisfiability Problem [7, Section 7.2.2.2] asks for an assignment of $n$ Boolean variables $x_{i} \in\{\mathrm{T}, \mathrm{F}\}$ (true, false), that satisfies a given Boolean formula expressed in Conjunctive Normal Form (CNF) e.g.

$$
\left(x_{1} \vee \neg x_{2}\right) \wedge\left(x_{2} \vee x_{3}\right) \wedge\left(\neg x_{1} \vee \neg x_{3}\right) \wedge\left(\neg x_{1} \vee \neg x_{2} \vee x_{3}\right) \wedge\left(x_{1} \vee x_{2} \vee \neg x_{3}\right)
$$

as a logical AND $(\wedge)$ of $m$ clauses $\mathcal{C}_{j}$, the expressions in parenthesis, each clause being composed by the logical OR $(\mathrm{V})$ of $k$ or less Boolean variables possibly preceded by logical NOT $(\neg)$; in this example $n=3, m=5, k=3$. A solution of the problem is either an assignment of $x_{i}$ that makes the expression $\mathrm{T}$ or a proof that such an assignment does not exist.

SAT was the first combinatorial problem proven to be NP-complete [5]; in particular while the case of $k=3$, 3SAT, can be solved only in a time that grows exponentially with $n, 2 \mathrm{SAT}$ and $1 \mathrm{SAT}$ problems can be solved in polynomial time (that is fast).

A 1SAT problem is just a logical AND of $m$ Boolean variables. For both assignments of $x_{i}, x_{i} \wedge \neg x_{i} \equiv \mathrm{F}$ and thus the presence of a variable together with its logical complement is a necessary and sufficient condition for making a 1SAT formula unsatisfiable. We use $\equiv$ for logical equivalence, namely that for all possible values taken by the variables the two expressions are equal, to mark the difference with algebraic equality $=$. We can interpret a satisfiable 1SAT formula as an assignment of variables since there is only one assignment that makes it $\mathrm{T}$ and that can be read scanning the formula; in the sequel we will freely use 1SAT formulas for assignments.

Using the distributive properties of the logical operators $\vee, \wedge$ any given $k \mathrm{SAT}$ formula expands in a logical OR of up to $k^{m}$ 1SAT terms this being just an upper bound since terms containing $x_{i} \wedge \neg x_{i}$ can be omitted. The final expanded expression is easily simplified and reordered exploiting the commutativity of the logical operators $\vee, \wedge$ and the properties $x_{i} \wedge x_{i} \equiv$ $x_{i} \vee x_{i} \equiv x_{i}$. It is easy to see that all "surviving" terms of this expansion, the Disjunctive Normal Form (DNF), are 1SAT terms, each of them representing an assignment that satisfies the problem. On the contrary if the DNF is empty, as happens for the example given above, this is a proof that no assignment can make the formula $\mathrm{T}$ : the problem is unsatisfiable.

Expansion to DNF is a terrible algorithm for SAT: first of all the method is an overkill since it produces all possible solutions whereas one is enough for SAT; in second place this brute force approach gives a running time proportional to the number of expansion terms $\mathcal{O}\left(\left(k^{\frac{m}{n}}\right)^{n}\right)$ whereas the present best SAT solvers [8] run in $\mathcal{O}\left(1.307^{n}\right)$. On the other hand DNF will assume a precise meaning in Clifford algebra.

We will write a SAT problem $\mathcal{S}$ in a more concise form as

$$
\mathcal{S} \equiv\left(\rho_{i} \vee \rho_{j} \vee \cdots \vee \rho_{h}\right)\left(\rho_{l} \vee \rho_{o} \vee \cdots \vee \rho_{p}\right) \cdots\left(\rho_{t} \vee \rho_{u} \vee \cdots \vee \rho_{z}\right) \quad \rho_{i} \in\left\{x_{i}, \bar{x}_{i}\right\}
$$


where $\rho_{i}$ is a literal that stands for Boolean variable $x_{i}$ or its complement, $\bar{x}_{i}$ for short, and the ordinary product stands for logical AND.

\section{Boolean expressions in Clifford algebra}

To tackle Boolean expressions in Clifford algebra we exploit the known fact that in any associative, unital, algebra every family of commuting, orthogonal, idempotents generates a Boolean algebra. This will allow us to put SAT problems in algebraic form. We proceed step by step along this path with SAT problems in mind.

Given a Boolean expression with $n$ Boolean variables $x_{i}$ we will represent it with an idempotent of the Clifford algebra $\mathcal{C} \ell\left(\mathbb{R}^{n, n}\right)$ that is isomorphic to the algebra of real matrices $\mathbb{R}\left(2^{n}\right)$. This algebra is more easily manipulated exploiting the properties of its Extended Fock Basis (EFB, see [2] and references therein) with which any algebra element is a linear superposition of simple spinors. The $2 n$ generators of the algebra $e_{i}$ form an orthonormal basis of the neutral vector space $V=\mathbb{R}^{n, n}$ with e.g.

$$
e_{i} e_{j}+e_{j} e_{i}:=\left\{e_{i}, e_{j}\right\}=2 \delta_{i j}(-1)^{i+1} \quad i, j=1,2, \ldots, 2 n
$$

while the Witt, or null, basis of $V$ is:

$$
\left\{\begin{array}{l}
p_{i}=\frac{1}{2}\left(e_{2 i-1}+e_{2 i}\right) \\
q_{i}=\frac{1}{2}\left(e_{2 i-1}-e_{2 i}\right)
\end{array} \quad i=1,2, \ldots, n\right.
$$

that, with $e_{i} e_{j}=-e_{j} e_{i}$, gives

$$
\left\{p_{i}, p_{j}\right\}=\left\{q_{i}, q_{j}\right\}=0 \quad\left\{p_{i}, q_{j}\right\}=\delta_{i j}
$$

showing that all $p_{i}, q_{i}$ are mutually orthogonal, also to themselves, that implies $p_{i}^{2}=q_{i}^{2}=0$, at the origin of the name "null" given to these vectors. The $2^{2 n}$ simple spinors forming EFB are given by all possible sequences

$$
\psi_{1} \psi_{2} \cdots \psi_{n} \quad \psi_{i} \in\left\{q_{i} p_{i}, p_{i} q_{i}, p_{i}, q_{i}\right\} \quad i=1, \ldots, n
$$

Since $e_{2 i-1} e_{2 i}=q_{i} p_{i}-p_{i} q_{i}:=\left[q_{i}, p_{i}\right]$ in EFB the identity $\mathbb{1}$ and the volume element $\omega$ (scalar and pseudoscalar) assume similar expressions [2]:

$$
\begin{aligned}
\mathbb{1} & :=\left\{q_{1}, p_{1}\right\}\left\{q_{2}, p_{2}\right\} \cdots\left\{q_{n}, p_{n}\right\} \\
\omega & :=e_{1} e_{2} \cdots e_{2 n}=\left[q_{1}, p_{1}\right]\left[q_{2}, p_{2}\right] \cdots\left[q_{n}, p_{n}\right] .
\end{aligned}
$$

With (4) and $q_{i} p_{i}+p_{i} q_{i}=1$ we easily obtain

$q_{i} p_{i} q_{i} p_{i}=q_{i} p_{i} \quad p_{i} q_{i} p_{i} q_{i}=p_{i} q_{i} \quad q_{i} p_{i} p_{i} q_{i}=p_{i} q_{i} q_{i} p_{i}=0 \quad q_{i} p_{i} q_{j} p_{j}=q_{j} p_{j} q_{i} p_{i}$ 
that shows that $q_{i} p_{i}$ and $p_{i} q_{i}$ are part of a family of orthogonal, commuting, idempotents. Since $\mathcal{C} \ell\left(\mathbb{R}^{n, n}\right)$ is a simple algebra, the unit element of the algebra is the sum of $2^{n}$ primitive (indecomposable) idempotents $\mathbb{p}_{i}$

$$
\mathbb{1}=\sum_{i=1}^{2^{n}} \mathbb{P}_{i}=\prod_{j=1}^{n}\left\{q_{j}, p_{j}\right\}
$$

where the second form, a product of $n$ anticommutators, is its expression in EFB (6). The full expansion of these anticommutators contains $2^{n}$ terms each term being one of the primitive idempotents and a simple spinor (5). We remind the standard properties of the primitive idempotents

$$
\mathbb{p}_{i}^{2}=\mathfrak{p}_{i} \quad\left(\mathbb{1}-\mathfrak{p}_{i}\right)^{2}=\mathbb{1}-\mathbb{p}_{i} \quad \mathfrak{p}_{i}\left(\mathbb{1}-\mathfrak{p}_{i}\right)=0 \quad \mathfrak{p}_{i} \mathbb{P}_{j}=\delta_{i j} \mathfrak{p}_{i} .
$$

Let $\mathcal{P} \subset \mathcal{C} \ell\left(\mathbb{R}^{n, n}\right)$ be the even, Abelian, subalgebra given by the linear space spanned by this family of $2^{n}$ primitive idempotents $\mathbb{p}_{i}$. In the isomorphic matrix algebra $\mathbb{R}\left(2^{n}\right) \mathcal{P}$ is usually, but not necessarily, the subalgebra of diagonal matrices and in this case the primitive idempotents are the $2^{n}$ diagonal matrices with a single 1 on the diagonal. Subalgebra $\mathcal{P}$ contains a subset in which field coefficients are binary, namely

$$
\mathcal{I}:=\left\{\sum_{i=1}^{2^{n}} \delta_{i} \mathbb{P}_{i}: \delta_{i} \in\{0,1\}\right\} \subset \mathcal{P}
$$

that is closed under multiplication but not under addition and is thus not even a subspace. With primitive idempotent properties (8) we can prove

Proposition 1. Given $s \in \mathcal{P}$ then $s \in \mathcal{I}$ if and only if $s$ is an idempotent, $s^{2}=s$

and thus $\mathcal{I}$ is the set of the idempotents, in general not primitive; a simple consequence is that for any $s \in \mathcal{I}$ also $(\mathbb{1}-s) \in \mathcal{I}$. $\mathcal{I}$ properties are easier to grasp observing that $\delta_{i} \in\{0,1\}$, the only idempotents of $\mathbb{R}$.

We remark that the $2^{n}$ primitive idempotents $\left\{\mathbb{p}_{i}\right\}$ form an orthonormal basis $^{1}$ of $\mathcal{P}$ as a linear space and thus uniquely identify all elements of $\mathcal{P}$ and thus of $\mathcal{I}(9)$ moreover by (8) the $i$-th component of $s \in \mathcal{P}$ is $\mathfrak{p}_{i} s$.

We are now ready to give the rules to replace Boolean elements with algebraic ones (using $\bar{\rho}_{i}$ for $\neg \rho_{i}$ )

$$
\begin{array}{ll}
\mathrm{F} & \rightarrow 0 \\
\mathrm{~T} & \rightarrow \mathbb{1} \\
\rho_{i} & \rightarrow q_{i} p_{i} \\
\bar{\rho}_{i} & \rightarrow \mathbb{1}-q_{i} p_{i} \\
\rho_{i} \wedge \rho_{j} & \rightarrow q_{i} p_{i} q_{j} p_{j}
\end{array}
$$

\footnotetext{
${ }^{1}$ not strictly a basis since $\mathfrak{p}_{i}^{2}=p_{i}$ but the set has all the other properties of an orthonormal basis
} 
the rules for the substitutions of the logical AND and NOT holding for any Boolean expression and not just for literals. With $\left\{p_{i}, q_{i}\right\}=\mathbb{1}$ and with De Morgan's relations we easily get

$$
\begin{array}{ll}
\bar{\rho}_{i} & \rightarrow p_{i} q_{i} \\
\rho_{i} \vee \rho_{j} & \rightarrow q_{i} p_{i}+q_{j} p_{j}-q_{i} p_{i} q_{j} p_{j}=\rho_{i}+\rho_{j}-\rho_{i} \rho_{j}
\end{array}
$$

where we take the liberty to use freely $\rho_{i}$ also in $\mathcal{C} \ell\left(\mathbb{R}^{n, n}\right)$ since it identifies with $q_{i} p_{i}$ with Clifford product standing for logical AND.

With a simple exercise we see that all elements of $\mathcal{C} \ell\left(\mathbb{R}^{n, n}\right)$ of (10) and (11) are idempotents, including the expression of the logical OR, and thus, by proposition 1 , are in $\mathcal{I}$. Since the set $\mathcal{I}$ is closed under multiplication also any $1 \mathrm{SAT}$ formula is in $\mathcal{I}$ and an idempotent. At this point it is manifest that the $2^{n}$ primitive idempotents of the basis $\left\{\mathbb{p}_{i}\right\}$ are in one to one correspondence with the possible $2^{n}$ 1SAT formulas of the $n$ literals (Boolean atoms), for example:

$$
\rho_{1} \bar{\rho}_{2} \cdots \rho_{n} \rightarrow q_{1} p_{1} p_{2} q_{2} \cdots q_{n} p_{n} .
$$

It is also instructive to derive that e.g. $q_{1} p_{1} \in \mathcal{I}$ directly from EFB formalism; with (7)

$$
q_{1} p_{1}=q_{1} p_{1} \mathbb{1}=q_{1} p_{1} \prod_{j=2}^{n}\left\{q_{j}, p_{j}\right\}
$$

since $q_{1} p_{1}\left\{q_{1}, p_{1}\right\}=q_{1} p_{1}$ and the full expansion is a sum of $2^{n-1} \mathrm{EFB}$ terms that are all primitive idempotents and thus $q_{1} p_{1} \in \mathcal{I}$ the sum being precisely the expansion in the basis $\left\{\mathbb{p}_{i}\right\}$. From the logical viewpoint this can be interpreted as the property that given the 1SAT formula $\rho_{1}$ the other, unspecified, $n-1$ literals $\rho_{2}, \ldots, \rho_{n}$ are free to take all possible $2^{n-1}$ values or, more technically, that the DNF $\rho_{1}$ has a full DNF with $2^{n-1}$ terms.

We prove now the general result that maps any Boolean expression with $n$ Boolean variables in $\mathcal{C} \ell\left(\mathbb{R}^{n, n}\right)$ :

Proposition 2. Any Boolean expression $\mathcal{S}$ with $n$ Boolean variables is represented in $\mathcal{C} \ell\left(\mathbb{R}^{n, n}\right)$ by $S \in \mathcal{I}$ by means of substitutions (10) moreover $\overline{\mathcal{S}}$ is represented by $\mathbb{1}-S$ both being idempotents. Given another Boolean expression $\mathcal{Q}$ the logical equivalence $\mathcal{S} \equiv \mathcal{Q}$ holds if and only if for their respective idempotents $S=Q$.

Proof. Any Boolean expression can be rewritten using only logical AND and NOT and thus we need only to check that (10) reproduces these two functions faithfully in $\mathcal{C} \ell\left(\mathbb{R}^{n, n}\right)$. So we verify that $\rho_{i} \wedge \rho_{j}$ is $\mathrm{F}$ if and only if, for the corresponding expression in $\mathcal{C} \ell\left(\mathbb{R}^{n, n}\right), \rho_{i} \rho_{j}=0$.

To do so we use the property that any Boolean expression $\mathcal{S}$ is $\mathrm{T}$ for a given assignment of its $n$ literals, expressed as a 1SAT formula, e.g. 
$\rho_{1} \rho_{2} \cdots \rho_{n}$, if and only if $\mathcal{S} \wedge \rho_{1} \rho_{2} \cdots \rho_{n}$ is $\mathrm{T}$ and we may thus interpret this formula as the substitution of $\rho_{1} \rho_{2} \cdots \rho_{n}$ into $\mathcal{S}$. Put in another way considering $\mathcal{S}$ in full DNF this formula selects term $\rho_{1} \rho_{2} \cdots \rho_{n}$ (if it is present) a kind of projection, a term that will turn out to be exact in $\mathcal{C} \ell\left(\mathbb{R}^{n, n}\right)$.

In $\mathcal{C} \ell\left(\mathbb{R}^{n, n}\right)$ for all possible substitutions for $\rho_{i} \rho_{j}$, namely $\rho_{i} \rho_{j}, \rho_{i} \bar{\rho}_{j}, \bar{\rho}_{i} \rho_{j}$ or $\bar{\rho}_{i} \bar{\rho}_{j}$, only the first one is non zero and thus the logical function AND is correctly mapped to Clifford product. Similarly for the logical NOT: to $\bar{\rho}_{i}$ corresponds $\mathbb{1}-\rho_{i}$ and by idempotent properties $\left(\mathbb{1}-\rho_{i}\right) \rho_{i}=0$ and, interpreting the first term as the expression of $\bar{\rho}_{i}$ in $\mathcal{C} \ell\left(\mathbb{R}^{n, n}\right)$ and the second as a substitution of Boolean variables, the proof is complete when we observe that $\rho_{i}$ is the only of the two possible assignments giving this result.

Starting from Boolean expression $\mathcal{S}$ written only with AND and NOT we see that the corresponding $S \in \mathcal{C} \ell\left(\mathbb{R}^{n, n}\right)$ obtained by (10) is in $\mathcal{I}$ since we already noticed that all literals are in $\mathcal{I}$ and that the set is closed under multiplication and under inner operation $s \rightarrow \mathbb{1}-s$.

For the part on logical equivalence $\mathcal{S} \equiv \mathcal{Q}$ we consider the expressions in their full DNF and we just remarked that $\mathcal{S} \wedge \rho_{1} \rho_{2} \cdots \rho_{n}$ selects the corresponding DNF term if it exists, thus logical equivalence holds if and only if they are equal term by term. With rules (10) we obtain two expressions $S, Q \in \mathcal{I}$ that have unique expansions (9) in the basis of primitive idempotents $\left\{\mathbb{p}_{i}\right\}$. By standard properties of the basis of a linear space, $S=Q$ is equivalent to the equality of their base expansions that can be checked component by component by $\mathrm{p}_{i} S=\mathrm{p}_{i} Q$ that is clearly equivalent to the termwise check of the full DNF of $\mathcal{S}$ and $\mathcal{Q}$.

We remark that the full equivalence between Boolean expressions and elements of $\mathcal{C} \ell\left(\mathbb{R}^{n, n}\right)$ holds only for elements of $\mathcal{I}$ that is not closed under addition so we will have to use algebra with some care. For example $S_{1}+S_{2}=$ $Q$ has a logical meaning if all terms belong to $\mathcal{I}$ and in this case this relation is equivalent to e.g. $S_{1}=Q-S_{2}$ to which we may attach a logical meaning, on the other hand $S_{1}+2 S_{2} \notin \mathcal{I}$ and strictly has no logical counterpart.

A useful property is that, for every $1 \leq i \leq n, \mathcal{P}$, as a vector space, may be written as a direct sum of two orthogonal subspaces (and subalgebras)

$$
\mathcal{P}=\mathcal{P}_{i} \oplus \overline{\mathcal{P}}_{i}=\rho_{i} \mathcal{P} \oplus \bar{\rho}_{i} \mathcal{P}
$$

where $\mathcal{P}_{i}$ and $\overline{\mathcal{P}}_{i}$ are subspaces of dimension $2^{n-1}$ obtained by projections $\mathcal{P}_{i}=q_{i} p_{i} \mathcal{P}=\rho_{i} \mathcal{P}$ as is simple to verify writing any element of $\mathcal{P}$ in $\left\{\mathbb{p}_{i}\right\}$ basis and remembering that $q_{i} p_{i}\left\{q_{i}, p_{i}\right\}=q_{i} p_{i}$, a projection already applied in (12). Both subspaces contain corresponding orthogonal subsets $\mathcal{I}_{i}$ and $\overline{\mathcal{I}}_{i}$ and, by (9), for any $s \in \mathcal{I}_{i}$ then $\mathbb{1}-s \in \overline{\mathcal{I}}_{i}$. 


\section{Satisifability in Clifford algebra}

Previous results apply to any Boolean expression and thus to SAT formulas but we give a more immediate way of expressing SAT problems in $\mathcal{C} \ell\left(\mathbb{R}^{n, n}\right)$ tailored to our needs:

Proposition 3. Given a SAT problem $\mathcal{S}(1)$ with $m$ clauses $\mathcal{C}_{j} \equiv\left(\rho_{j_{1}} \vee\right.$ $\left.\rho_{j_{2}} \vee \cdots \vee \rho_{j_{k}}\right)$, for each clause let $z_{j}:=\bar{\rho}_{j_{1}} \bar{\rho}_{j_{2}} \cdots \bar{\rho}_{j_{k}}$, then $\mathcal{S}$ is satisfiable if and only if, the corresponding algebraic expression of $\mathcal{C} \ell\left(\mathbb{R}^{n, n}\right)$

$$
S=\prod_{j=1}^{m}\left(\mathbb{1}-z_{j}\right) \neq 0 .
$$

Proof. With De Morgan's relations and (10) we get that clause $\mathcal{C}_{j}$ is represented in $\mathcal{C} \ell\left(\mathbb{R}^{n, n}\right)$ by

$$
\mathcal{C}_{j} \rightarrow \mathbb{1}-\bar{\rho}_{j_{1}} \bar{\rho}_{j_{2}} \cdots \bar{\rho}_{j_{k}}=\mathbb{1}-z_{j}
$$

and by previous results $\mathcal{C}_{j}$ is $\mathrm{F}$ for all and only the assignments giving $\mathbb{1}-z_{j}=0$ in $\mathcal{C} \ell\left(\mathbb{R}^{n, n}\right)$.

By the substitution mechanism mentioned in the proof of proposition 2 for all and only the assignments $\rho_{1} \rho_{2} \cdots \rho_{n}$ that satisfy $\mathcal{S}$ then $\mathcal{S} \wedge \rho_{1} \rho_{2} \cdots \rho_{n}$ is $\mathrm{T}$ and thus, necessarily, $\mathcal{C}_{j} \wedge \rho_{1} \rho_{2} \cdots \rho_{n}$ is $\mathrm{T}$ for all $j$. In $\mathcal{C} \ell\left(\mathbb{R}^{n, n}\right)$ this is equivalent to $\left(\mathbb{1}-z_{j}\right) \rho_{1} \rho_{2} \cdots \rho_{n}=\rho_{1} \rho_{2} \cdots \rho_{n} \neq 0$ for all $j$ and thus also $S \rho_{1} \rho_{2} \cdots \rho_{n} \neq 0$.

We remark that expanding the product of two clauses of (14) we get

$$
\left(\mathbb{1}-z_{j}\right)\left(\mathbb{1}-z_{l}\right)=\mathbb{1}-z_{j}-z_{l}+z_{j} z_{l}
$$

and $z_{j} z_{l}=0$ if and only if in $z_{j}$ and $z_{l}$ appears the same literal in opposite forms $\left(\rho_{i} \bar{\rho}_{i}=0\right)$. In any way this product is always in $\mathcal{I}$ even if the generic terms of the expansion are not in general in $\mathcal{I}$, e.g. $-z_{j}$. In general the product of $m$ clauses will expand to at most $2^{m}$ terms that is nothing else than the Boolean expansion of $\mathcal{S}$ in its DNF of section 2 and the expansion of $S$ in basis $\left\{\mathbb{p}_{i}\right\}$. The first term of the expansion is certainly $\mathbb{1}$ (15) so that, calling $\Delta$ the other terms we rewrite (14) as

$$
S=\prod_{j=1}^{m}\left(\mathbb{1}-z_{j}\right):=\mathbb{1}-\Delta
$$

and since $S \in \mathcal{I}$ also $\Delta=\mathbb{1}-S$ is in $\mathcal{I}$ and all elements of this relation are idempotents. 


\section{A condition for unsatisfiability}

Exploiting this SAT formulation we prove the main result of this paper:

Theorem 1. A given nonempty $S A T$ problem in $\mathcal{C} \ell\left(\mathbb{R}^{n, n}\right)$ (14) admits no solution if and only if, for all generators (2) of $\mathcal{C} \ell\left(\mathbb{R}^{n, n}\right)$

$$
e_{i} S e_{i}^{-1}=S \quad \forall 1 \leq i \leq 2 n .
$$

Proof. We know that $S \in \mathcal{I}$; a simple consequence of proposition 5 in next section is that also $e_{i} S e_{i}^{-1} \in \mathcal{I}$ so both terms are in $\mathcal{I}$.

By (16) $e_{i} S e_{i}^{-1}=S$ if and only if $e_{i} \Delta e_{i}^{-1}=\Delta$ and by a fundamental property of Clifford algebra [9, Propostion 16.6] an element of the even subalgebra commutes with all generators $e_{i}$ if and only it is of the form $\delta \mathbb{1}$ for $\delta \in \mathbb{R}$. So $e_{i} \Delta e_{i}^{-1}=\Delta$ for all $i$ is equivalent to $\Delta=\delta \mathbb{1}$ but we know also that $\Delta \in \mathcal{I}$ and thus necessarily $\delta \in\{0,1\}$. By (16) $\delta=0$ would imply $S=\mathbb{1}$ that represents a SAT problem with no clauses, excluded by hypothesis, so $\Delta=\mathbb{1}$ that implies $S=0$ that is thus unsatisfiable.

It is known that a given SAT problem $\mathcal{S}$ is unsatisfiable if and only if $\overline{\mathcal{S}}$ is a tautology, namely if $\overline{\mathcal{S}} \equiv \mathrm{T}$. By proposition 2 and (16) $\bar{S}=\mathbb{1}-S=\Delta$ that gives the algebraic version of this property in $\mathcal{I}$ and we have

Corollary 4. A Boolean expression $\overline{\mathcal{S}}$ is a tautology if and only if for its corresponding idempotent $\Delta \in \mathcal{I}, \Delta \neq 0$ and

$$
e_{i} \Delta e_{i}^{-1}=\Delta \quad \forall 1 \leq i \leq 2 n
$$

and thus $\mathbb{1} \in \mathcal{I}$ represent a tautology and (7) is its full DNF.

We remark that in $\mathcal{C} \ell\left(\mathbb{R}^{n, n}\right)$ tautologies and unsatisfiable SAT, corresponding respectively to $S=\mathbb{1}, 0$, are the only instances of SAT that have the maximally symmetric form of the scalar elements of $\mathcal{C} \ell\left(\mathbb{R}^{n, n}\right)(17)$.

\section{An algorithm to test for unsatisfiability}

We exploit results of previous section to outline a SAT algorithm: its basic idea is that if a given SAT problem is satisfiable it infringes at least one of the $2 n$ symmetry relations of theorem 1 . We begin with a simple result:

Proposition 5. For any literal $\rho_{i}$ in $\mathcal{C} \ell\left(\mathbb{R}^{n, n}\right)$

$$
\begin{array}{lll}
e_{j} \rho_{i} e_{j}^{-1}=\bar{\rho}_{i} & \text { for } & j \in\{2 i-1,2 i\} \\
e_{j} \rho_{i} e_{j}^{-1}=\rho_{i} & \text { for } & j \notin\{2 i-1,2 i\}
\end{array}
$$

proved using (2) and (3). Thus, when applying theorem 1, it is sufficient to test invariance just for the odd generators $e_{2 i-1}$ for which $e_{2 i-1}^{-1}=e_{2 i-1}$ and 
the literal $\rho_{i}$ is complemented by "its" generator $e_{2 i-1}$ and left invariant by all others, a typical case of reflections in Clifford algebras.

With this result we can check theorem 1 in a simple example with $n=$ 1: the only unsatisfiable SAT, $\rho_{1} \bar{\rho}_{1}$, is actually invariant since $e_{1} \rho_{1} \bar{\rho}_{1} e_{1}=$ $\rho_{1} \bar{\rho}_{1}$. The theorem now looks sensible also in the Boolean formulation of SAT: a problem is unsatisfiable if and only if exchanging any literal for its complement the result is not altered.

To derive an actual algorithm from these relations we need a technical

Lemma 1. Given a non empty $k S A T$ problem $S$ with $n$ literals, for any literal $\rho_{i}$ we may write $S$ as

$$
S=\bar{\rho}_{i} S_{\underline{i} 1}+\rho_{i} S_{\underline{i} 2}
$$

where $S_{\underline{1} 1}$ and $S_{\underline{i} 2}$ are $S A T$ problems, represented in $\mathcal{I}$, with $n-1$ literals: all those of $S$ but the $i$-th, this being the reason for notation $\underline{i}$. More in detail:

$$
S_{\underline{i} 1}=S_{\underline{0} 0} S_{\underline{i} 1}^{\prime} \quad S_{\underline{i} 2}=S_{\underline{i} 0} S_{\underline{i} 2}^{\prime}
$$

where:

- $S_{\underline{i} 0}$ is the $k S A T$ problem formed by the subset of clauses of $S$ that do not contain neither $\rho_{i}$ nor $\bar{\rho}_{i}$;

- $S_{\underline{1}}^{\prime}$ is the $(k-1) S A T$ problem formed by the subset of clauses of $S$ that contained $\bar{\rho}_{i}$ and in which $\bar{\rho}_{i}$ have been removed;

- $S_{\underline{2} 2}^{\prime}$ is the $(k-1) S A T$ problem formed by the subset of clauses of $S$ that contained $\rho_{i}$ and in which $\rho_{i}$ have been removed.

Proof. We have seen that for any literal $\rho_{i}$ we can decompose $\mathcal{P}$ in a direct sum of orthogonal subspaces (13), it follows that any $S \in \mathcal{I}$ has a unique decomposition with components belonging to the two subspaces; the components are obtained by projections $\bar{\rho}_{i} S$ and $\rho_{i} S$ so that $S=\bar{\rho}_{i} S+\rho_{i} S$, moreover both terms are in $\mathcal{I}$. Calculating explicitly the terms of (18) with the projections is immediate with $S$ in the form (14).

Proposition 6. When testing the invariance of a given Boolean expression $S$ for any single generator $e_{2 i-1}$ for $1 \leq i \leq n$ the three following statements are equivalent

$$
\begin{aligned}
e_{2 i-1} S e_{2 i-1} & =S \\
S_{\underline{1} 1} & =S_{\underline{i} 2} \\
S & =\left(\rho_{i}+\bar{\rho}_{i}\right) S_{\underline{1} 1}=S_{\underline{i} 1}
\end{aligned}
$$

with $S_{\underline{1} 1}$ and $S_{\underline{i} 2}$ defined as in lemma 1 . 
Proof. To prove the proposition we prove that any statement implies the successive, circularly. Along the proof we will see that all terms of these relations are in $\mathcal{I}$ and so the use of algebraic equality is consistent.

Assuming the first statement and writing $S$ in form (18) we get

$$
e_{2 i-1}\left(\bar{\rho}_{i} S_{\underline{i} 1}+\rho_{i} S_{\underline{i} 2}\right) e_{2 i-1}=\bar{\rho}_{i} S_{\underline{i} 2}+\rho_{i} S_{\underline{1} 1}
$$

since $S_{\underline{1} 1}$ and $S_{\underline{2} 2}$ are without the $i$-th literal and thus, by proposition 5 , are left invariant by $e_{2 i-1}$. The two terms belong to the two orthogonal subspaces $\rho_{i} \mathcal{P}$ and $\bar{\rho}_{i} \mathcal{P}$ of $\mathcal{P}(13)$. It follows that equality with $\bar{\rho}_{i} S_{\underline{i} 1}+\rho_{i} S_{\underline{i} 2}$ holds only if it holds separately in the two subspaces and this happens iff

$$
S_{\underline{i} 1}=S_{\underline{i} 2}
$$

Assuming this relation with $S$ in form (18) we immediately get with (10)

$$
S=\left(\rho_{i}+\bar{\rho}_{i}\right) S_{\underline{1} 1}=S_{\underline{1} 1}
$$

and in turn this form guarantees $e_{2 i-1} S e_{2 i-1}=S$.

With these results we outline a recursive algorithm for $k \mathrm{SAT}$ problem $S$ : we select a literal $\rho_{i}$, calculate $S$ in form (18) and test wether $S_{\underline{i} 1}=S_{\underline{i} 2}{ }^{2}$. If the answer is no by theorem 1 and proposition 6 we have a certificate that $S$ is satisfiable. If the answer is yes by proposition 6 we can write $S=S_{\underline{i} 1}$ and we restart with this new problem in $n-1$ literals. If the process continues for all the $n$ literals we fulfill the conditions of theorem 1 proving that $S$ has the fully symmetric form of the scalars $(7)$ in $\mathcal{C} \ell\left(\mathbb{R}^{n, n}\right)$ and is thus unsatisfiable.

In practice it can be difficult to prove or disprove that $S_{\underline{i} 1}=S_{i 2}$ and we need to use a slightly more complex strategy based on the following principle: $S_{\underline{1} 1}=S_{\underline{i} 2}$ is equivalent to $S_{\underline{i} 1}-S_{\underline{2} 2}=0$ and since this is an algebraic expression in $n-1$ literals we can check that it is a fully symmetric expression, namely a scalar in Clifford algebra $\mathcal{C} \ell\left(\mathbb{R}^{n-1, n-1}\right)$.

We conclude showing that this algorithm constitutes a relaxation of the Davis Putnam recursive SAT algorithm (DPLL) based on results we resume here, in our notation to ease comparisons [6, Theorem 4.1 p. 243]:

1. any $k \mathrm{SAT}$ problem $\mathcal{S}$ can be written, with respect to any of its literals $\rho_{i}$, in the form (lemma 1 gives an alternative algebraic proof of this)

$$
\mathcal{S} \equiv \bar{\rho}_{i} \mathcal{S}_{\underline{i} 1} \vee \rho_{i} \mathcal{S}_{\underline{i} 2}
$$

\footnotetext{
${ }^{2}$ we remark that using the more detailed version of (18) $S=\bar{\rho}_{i} S_{\underline{i} 0} S_{\underline{1}}^{\prime}+\rho_{i} S_{\underline{i} 0} S_{\underline{i 2}}^{\prime}$ if $S_{\underline{i} 0}$ is invertible condition $S_{\underline{i} 0} S_{\underline{i} 1}^{\prime}=S_{\underline{\underline{0}}} S_{\underline{i 2}}^{\prime}$ is equivalent to $S_{\underline{i} 1}^{\prime}=S_{\underline{i 2}}^{\prime}$ that is usually a much simpler problem since $\bar{S}_{\underline{i} 1}^{\prime}$ and $S_{\underline{i}}^{\bar{\prime}}$ are $(k-1)$ SAT problems. But $S_{\underline{i} 0} \in \mathcal{I}$ and thus it is invertible only if $S_{\underline{i} 0}=\mathbb{1}$, namely if $S_{\underline{i} 0}$ has no clauses. Nevertheless the condition $S_{\underline{i} 1}^{\prime}=S_{\underline{i} 2}^{\prime}$ is sufficient to get $S_{\underline{\underline{0}} 0} S_{\underline{i} 1}^{\prime}=S_{\underline{i} 0} S_{\underline{i}}^{\prime}$ but in general not necessary.
} 
2. $\mathcal{S}$ is unsatisfiable if and only if for all literals $\rho_{i}$ the derived problems $\mathcal{S}_{\underline{i} 1}$ and $\mathcal{S}_{\underline{i} 2}$ are both unsatisfiable $\mathcal{S}_{\underline{i} 1} \equiv \mathcal{S}_{\underline{i} 2} \equiv \mathrm{F}$, in our notation

$$
S_{\underline{i} 1}=S_{\underline{i} 2}=0 \text {. }
$$

These properties are at the heart of a recursive algorithm very similar to our one with the noticeable difference that condition (20) is stronger than (19) and in this sense our algorithm is a relaxation of DPLL.

In other words $S_{\underline{1} 1}=S_{\underline{i} 2} \neq 0$ does not satisfy (20) but satisfies (19) and we can proceed to test invariance with respect to the next literal. Only if the procedure continues up to the last literal, by theorem 1 , we will have proved that $S$ is a scalar and thus $S=0$ and so only if we arrive at the end we will have proved that at every step we actually had not only $S_{\underline{1} 1}=S_{\underline{i} 2}$ but $S_{\underline{i} 1}=S_{\underline{i} 2}=0$. A more detailed version of this algorithm together with a formal proof of its correctness is contained in a dedicated appendix.

\section{Conclusions}

It is not clear wether these results can produce an algorithm competitive with state of the art SAT solvers but its theoretical properties look interesting at least for the transformation of a typical combinatorial problem, it is tempting to say the archetypical combinatorial problem, in a purely algebraic setting in Clifford algebra.

The main contribution of Clifford algebra is that it allows to deal with the exponentially large DNF expressions of SAT problems algebraically. Moreover testing unsatisfiability by means of geometric invariance of idempotents under reflections of $e_{2 i-1}$ generators appears promising.

SAT formulation (16) can be exploited to find also other conditions guaranteeing unsatisfiability, namely $\bar{S}=\Delta=\mathbb{1}$. For example in the isomorphic matrix algebra $\mathbb{R}\left(2^{n}\right), \Delta$ can be always a diagonal matrix and thus the problem is satisfiable if and only if $\operatorname{det}(\Delta)=0$. With Clifford algebra we can manipulate these matrices of size $2^{n}$ manipulating just $n$ matrices of size 2 , e.g. with Jordan-Wigner construction.

We conclude noting that Clifford algebra, beyond its ability to represent automorphisms of linear spaces, is well suited also to deal with Boolean expressions.

\section{Appendix}

In section 6 we outlined a SAT algorithm that exploits the relaxation of (20) to (19): we describe it here together with a proof of its correctness.

We start proving two propositions that extend theorem 1: the first one to milder conditions and the second one to generalized variables. 
Proposition 7. A non empty SAT problem $S$ is unsatisfiable if and only if for all literals $\rho_{i}$ and their relative expansion (18)

$$
S_{\underline{1} 1}-S_{\underline{i} 2}=\delta \mathbb{1} \quad \delta \in \mathbb{R} \quad \forall 1 \leq i \leq n .
$$

Proof. Assuming $S=0$ by theorem 1 and proposition 6 for any literal $\rho_{i}$ we have $S_{\underline{1} 1}-S_{\underline{i} 2}=0$. To prove the converse let $S_{\underline{i} 1}-S_{\underline{2} 2}=\delta \mathbb{1}$ with $\delta \in \mathbb{R}$; since both $S_{\underline{i} 1}$ and $S_{\underline{i} 2}$ are in $\mathcal{I}$ and belong to a $2^{n-1}$-dimensional subspace of $\mathcal{P}(13)$ we easily see that equality can hold only for $\delta=0$, thus $S_{\underline{i} 1}=S_{\underline{i} 2}$ and since this holds for all literals $\rho_{i}$, by theorem $1, S=0$.

To deal with expressions like $S_{\underline{i} 1}-S_{\underline{i} 2}$ in full generality we introduce a new kind of variables $\mathbb{S}$ of the form

$$
\mathbb{S}=\sum_{j=1}^{r} s_{j} S_{j} \quad s_{j}= \pm 1, S_{j} \in \mathcal{I}
$$

where $S_{j}$ are SAT problems with $n$ literals. In general $S_{j} \in \mathcal{I}$ and $\mathbb{S} \in \mathcal{P}$, being a sum of signed idempotents, but in general $\mathbb{S} \notin \mathcal{I}$. Also to $\mathbb{S}$ we may apply decomposition (18), applying it first to the SAT problems of the sum $S_{j}$ getting, with straightforward extension of notation,

$$
\mathbb{S}=\sum_{j=1}^{r} s_{j}\left(\bar{\rho}_{i} S_{j \underline{i} 1}+\rho_{i} S_{j \underline{i 2}}\right):=\bar{\rho}_{i} \mathbb{S}_{\underline{i} 1}+\rho_{i} \mathbb{S}_{\underline{i} 2}
$$

where we defined two new variables of the new kind, in $n-1$ literals

$$
\mathbb{S}_{\underline{i} 1}=\sum_{j=1}^{r} s_{j} S_{j \underline{i} 1} \quad \mathbb{S}_{\underline{i} 2}=\sum_{j=1}^{r} s_{j} S_{j \underline{i} 2} .
$$

Now we extend theorem 1 to the new variables $\mathbb{S}$ proving the slightly weaker relation $\mathbb{S}=\delta \mathbb{1}$, with $\delta \in \mathbb{R}$.

Proposition 8. Give a signed sum $\mathbb{S}$ of $r$ SAT problems in n literals (22) then $\mathbb{S}=\delta \mathbb{1}$ with $\delta \in \mathbb{R}$ if and only if, for all its literals $\rho_{i}$ we have in (23)

$$
\mathbb{S}_{\underline{i} 1}-\mathbb{S}_{\underline{i} 2}=0 \quad \forall 1 \leq i \leq n
$$

where each expression $\mathbb{S}_{\underline{1} 1}-\mathbb{S}_{\underline{i} 2}$ has at most $2 r$ terms in $n-1$ literals.

Proof. For $r=1$ previous results prove the thesis; for $r>1$ we remark that theorem 1, being a general property of Clifford algebra, applies to any element of $\mathcal{P}$ and thus to $\mathbb{S}$ and thus we can proceed with algebraic equality.

Given $\mathbb{S}$ we select a literal $\rho_{i}$ and apply the decomposition (23) and with proposition 5 we get

$$
e_{2 i-1} S_{2 i-1}=\bar{\rho}_{i} \Im_{\underline{i} 2}+\rho_{i} \Im_{\underline{i} 1}
$$

to be compared with (23); since we are in $\mathcal{P}$ by (13) the comparison breaks up into two parts in each subspace that have to be satisfied separately and this happens if and only if $\mathbb{S}_{\underline{i} 1}=\mathbb{S}_{\underline{i} 2}$. 
With these two results we proceed to build a recursive algorithm; we start building a chain of implications deriving from the hypothesis that a given nonempty SAT problem $S$ is unsatisfiable, namely $S=0$ : given any literal $\rho_{i}$, we have the necessary condition (21), namely

$$
S=0 \stackrel{\rho_{i}}{\Longrightarrow} \quad S_{\underline{i 1}}-S_{\underline{i} 2}=\delta \mathbb{1}
$$

where $\rho_{i}$ indicates that to go from the $n$ literals of $S$ to the $n-1$ of $S_{\underline{1} 1}-S_{\underline{i} 2}$ we eliminated the $i$-th literal. This is just an implication since to prove that $S=0$ we would need to prove the other $n-1$ similar implications, on the other hand $S_{\underline{1} 1}-S_{\underline{i} 2} \neq \delta \mathbb{1}$ proves that $S \neq 0$. The same argument can be applied to the signed sum of SAT problems $(22)$ in $\mathcal{P}$ :

$$
\mathbb{S}=\delta \mathbb{1} \quad \stackrel{\rho_{j}}{\Longrightarrow} \quad \mathbb{S}_{\underline{j} 1}-\mathbb{S}_{\underline{j} 2}=0 \quad \Longrightarrow \quad \mathbb{S}_{\underline{j} 1}-\mathbb{S}_{\underline{j} 2}=\delta \mathbb{1} .
$$

We can connect (24) and (25) and starting from a single SAT problem $S$ in $n$ literals we can build a complete chain of implications

$S=0 \stackrel{\rho_{1}}{\Longrightarrow} \mathbb{S}_{11}-\mathbb{S}_{12}=\delta \mathbb{1} \quad \stackrel{\rho_{2}}{\Longrightarrow} \mathbb{S}_{\underline{21}}-\mathbb{S}_{\underline{2} 2}=\delta \mathbb{1} \stackrel{\rho_{3}}{\Longrightarrow} \cdots \stackrel{\rho_{n}}{\Longrightarrow} \mathbb{S}_{\underline{n} 1}=\mathbb{S}_{\underline{n} 2}$

where in the last step $\rho_{n}$ represent the last literal to be eliminated and thus the rightmost expression of the chain has no literals and can be easily checked for equality since at the last stage the problems can be only of two kinds: unsatisfiable problems, namely problems with empty clauses, or tautologies, namely problems with no clauses and so the final comparison $\mathbb{S}_{\underline{n} 1}=\mathbb{S}_{\underline{n} 2}$ can be done immediately. Obviously the choice of the literal at each step is free.

We conclude proving that the satisfaction of the $n-1$ rightmost tests of (26), namely,

$$
S \stackrel{\rho_{1}}{\Longrightarrow} \mathbb{S}_{\underline{1} 1}-\mathbb{S}_{\underline{1} 2}=\delta \mathbb{1} \quad \stackrel{\rho_{2}}{\Longrightarrow} \quad \mathbb{S}_{\underline{2} 1}-\mathbb{S}_{\underline{2} 2}=\delta \mathbb{1} \quad \stackrel{\rho_{3}}{\Longrightarrow} \cdots \stackrel{\rho_{n}}{\Longrightarrow} \quad \mathbb{S}_{\underline{n} 1}=\mathbb{S}_{\underline{n} 2}
$$

implies in turn $S=0$ transforming the chain of implications (26) in a closed circle.

Proposition 9. Given a non empty SAT problem $S$ the implication list (27) is fully satisfied if and only if $S=0$.

Proof. The forward implication chain (26) have already been proved. To prove the converse we prove first that if any $\mathbb{S}_{\underline{i 1}}-\mathbb{S}_{\underline{i 2}} \neq \delta \mathbb{1}$, namely if the chain is broken, this implies that $S$ is satisfiable: this is immediate since $\mathbb{S}_{\underline{i} 1}-\mathbb{S}_{\underline{i} 2} \neq \delta \mathbb{1}$ implies $\mathbb{S}_{\underline{i} 1} \neq \mathbb{S}_{\underline{i} 2}$ and thus that our problem $S$ was not symmetric with respect to the just eliminated literal and by proposition 8 $S \neq \delta \mathbb{1}$ and it is satisfiable.

To prove that $\mathbb{S}_{\underline{n} 1}=\mathbb{S}_{\underline{n} 2}$ for the last expression of (27) implies $S=0$ we start from the last step where we can check easily if $\mathbb{S}_{\underline{n} 1}=\mathbb{S}_{\underline{n} 2}$ and in both 
cases (unsatisfiable or tautology) the expression is fully symmetric in all (none actually) the literals and so if $\mathbb{S}_{\underline{n} 1}=\mathbb{S}_{\underline{n} 2}$ we also have $\mathbb{S}_{\underline{n} 1}=\mathbb{S}_{\underline{n} 2}=\delta \mathbb{1}$ with $\delta \in\{0,1\}$.

We show that the property of being fully symmetric of the expression at hand propagates "upstream" along the implication chain (27) and when reaches the first element of the chain proofs that $S=\delta \mathbb{1}$.

Applying to any $\mathbb{S}_{\underline{i} 1}-\mathbb{S}_{\underline{i} 2}$ with $n^{\prime}$ literals recursion (23) with respect to literal $\rho_{j}$ we get, with heavy but hopefully clear notation,

$\mathbb{S}_{\underline{i} 1}-\mathbb{S}_{\underline{i} 2}=\bar{\rho}_{j} \mathbb{S}_{i j 11}+\rho_{j} \mathbb{S}_{\underline{i j 12}}-\bar{\rho}_{j} \mathbb{S}_{i j 21}-\rho_{j} \mathbb{S}_{i \underline{j} 22}=\bar{\rho}_{j}\left(\mathbb{S}_{\underline{i j 11}}-\mathbb{S}_{\underline{i j 21}}\right)+\rho_{j}\left(\mathbb{S}_{\underline{i j 12}}-\mathbb{S}_{i \underline{j} 22}\right)$

and we go at the next right level of (26) bringing the expression with $n^{\prime}-1$ literals

$$
\left(\mathbb{S}_{\underline{i j 11}}-\mathbb{S}_{\underline{i j 21}}\right)-\left(\mathbb{S}_{\underline{i j 12}}-\mathbb{S}_{\underline{i j 22}}\right)=\delta \mathbb{1} .
$$

If at the next level holds not only this relation but also the stronger relation $\left(\mathbb{S}_{i j 11}-\mathbb{S}_{\underline{i j 21}}\right)=\left(\mathbb{S}_{\underline{i j 12}}-\mathbb{S}_{i j 22}\right)=\delta \mathbb{1}$ with $\delta \in\{0,1\}$ this implies that the two expressions are necessarily fully symmetric in their $n^{\prime}-1$ literals, and this implies in turn that also for the expression at the previous step (28)

$$
\mathbb{S}_{\underline{i} 1}-\mathbb{S}_{\underline{i} 2}=\left(\bar{\rho}_{j}+\rho_{j}\right)\left(\mathbb{S}_{\underline{i j 11}}-\mathbb{S}_{\underline{i j} 21}\right)=\delta \mathbb{1} \quad \delta \in\{0,1\}
$$

and thus that $\mathbb{S}_{\underline{i} 1}-\mathbb{S}_{\underline{i 2}}$ is fully symmetric in all its $n^{\prime}$ literals. We conclude that this property propagates upstream the implication chain (27) until it reaches the first level, just after $S$ and, with proposition $7, S=0$.

This implication chain defines recursive algorithm 1 that, starting from $S$ eliminates one literal at the time producing a list of sum of signed SAT problems (22) that, by proposition 8 , at the last step can have $r \leq 2^{n}$ SAT problems. At each step we easily see that each $S_{j}=0$ can be eliminated from $\mathbb{S}$ and that each satisfiable $S_{j}$, being not fully symmetric, proves that the initial problem $S$ is satisfiable.

We remark that if in the running of the recursive algorithm $\mathbb{S}$ contains terms $S_{j}$ and $-S_{j}$ they can not be "simplified" since if $S_{j}$ is satisfiable, namely $S_{j} \neq 0$, replacing $S_{j}-S_{j}$ with 0 would replace a non fully symmetric term with a fully symmetric one, thus hiding a source of asymmetry (while the simplification can be done in a non recursive algorithm). A very preliminary version of the algorithm written in Mathematica solves correctly random SAT problems with tens of literals. A crude analysis of the running time, assuming recursion relation $A(n)=2 A(n-1)$, gives obviously $A(n)=\mathcal{O}\left(2^{n-1}\right)$. 


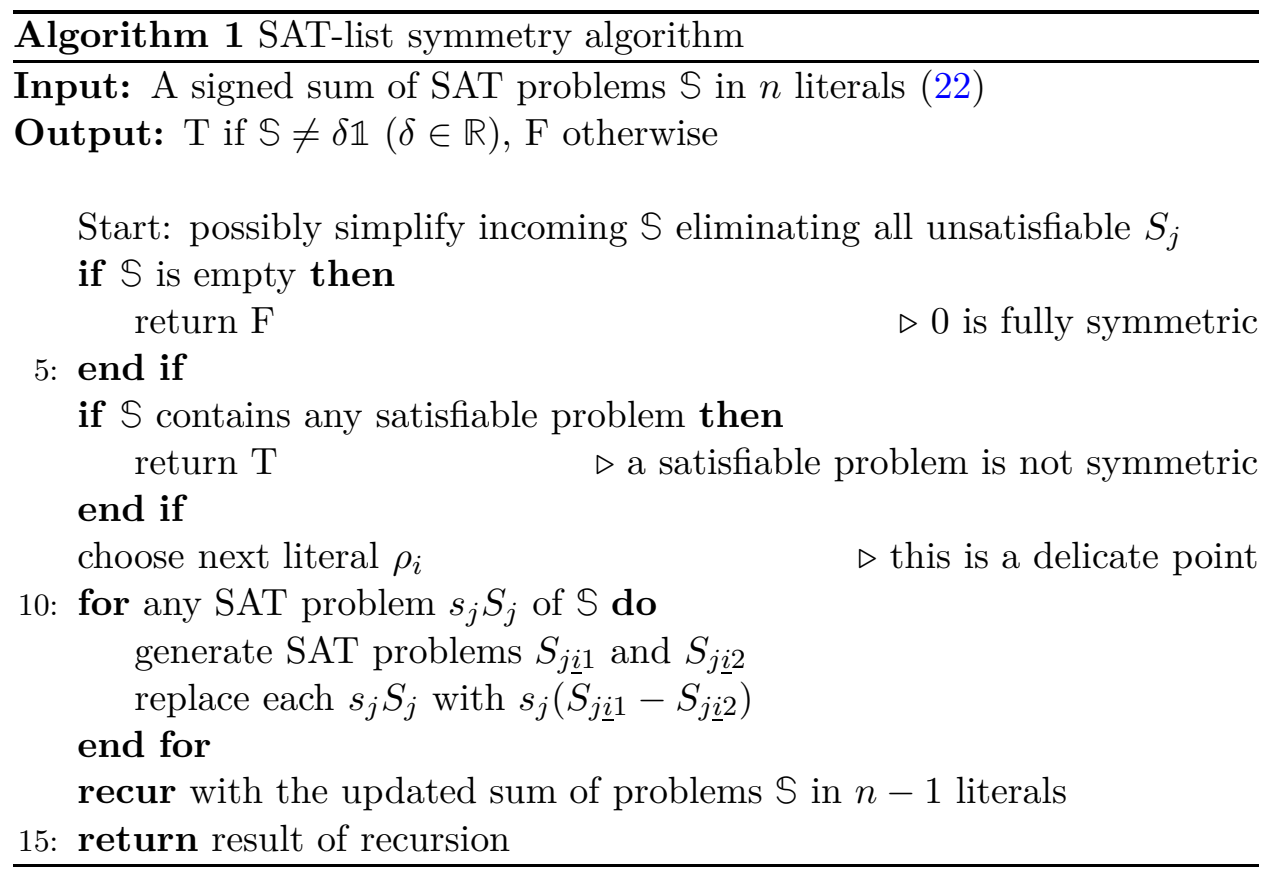




\section{References}

[1] Alfredo Braunstein, Marc Mézard, and Riccardo Zecchina. Survey propagation: an algorithm for satisfiability. Random Structures $\mathcal{E}$ Algorithms, 27(2):201-226, Nov 2005.

[2] Marco Budinich. On spinors transformations. Journal of Mathematical Physics, 57(7):071703-1-11, July 2016. arXiv:1603.02181 [math-ph] 7 Mar 2016.

[3] Élie Cartan. Les groupes projectifs qui ne laissent invariante aucune multiplicité plane. Bulletin de la Société Mathématique de France, 41:5396, 1913.

[4] Claude C. Chevalley. Algebraic Theory of Spinors. Columbia University Press, New York, 1954.

[5] Stephen A. Cook. The Complexity of Theorem-proving Procedures. In Proceedings of the Third Annual ACM Symposium on Theory of Computing, STOC '71, pages 151-158, New York, NY, USA, 1971. ACM.

[6] Martin David Davis and Elaine J. Weyuker. Computability, Complexity, and Languages. Academic Press, Boston, MA, 1983.

[7] Donald Ervin Knuth. The Art of Computer Programming. Combinatorial Algorithms, volume IV. Addison-Wiley, Reading, MA, release in fascicles edition, 2015.

[8] Ramamohan Paturi, Pavel Pudlák, Michael E. Saks, and Francis Zane. An Improved Exponential-time Algorithm for k-SAT. Journal of the $A C M, 52(3): 337-364$, May 2005.

[9] Ian Robertson Porteous. Clifford Algebras and the Classical Groups. Cambridge Studies in Advanced Mathematics 50. Cambridge University Press, 1995. 